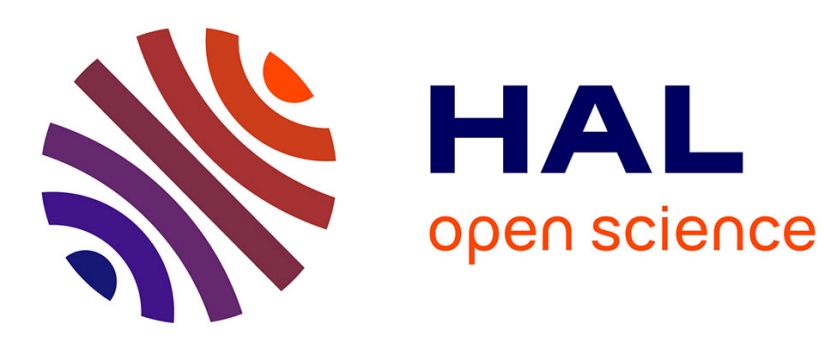

\title{
Toward a generalized tensegrity model describing the mechanical behaviour of the cytoskeleton structure
}

\author{
Sylvie Wendling-Mansuy, Patrick Cañadas, Patrick Chabrand
}

\section{To cite this version:}

Sylvie Wendling-Mansuy, Patrick Cañadas, Patrick Chabrand. Toward a generalized tensegrity model describing the mechanical behaviour of the cytoskeleton structure. Computer Methods in Biomechanics and Biomedical Engineering, 2003, 6 (1), pp.45-52. 10.1080/1025584021000059413 . hal-00088275

\section{HAL Id: hal-00088275 \\ https://hal.science/hal-00088275}

Submitted on 2 Jun 2020

HAL is a multi-disciplinary open access archive for the deposit and dissemination of scientific research documents, whether they are published or not. The documents may come from teaching and research institutions in France or abroad, or from public or private research centers.
L'archive ouverte pluridisciplinaire HAL, est destinée au dépôt et à la diffusion de documents scientifiques de niveau recherche, publiés ou non, émanant des établissements d'enseignement et de recherche français ou étrangers, des laboratoires publics ou privés. 


\title{
Toward a Generalised Tensegrity Model Describing the Mechanical Behaviour of the Cytoskeleton Structure
}

\author{
SYLVIE WENDLING ${ }^{\mathrm{a}, \mathrm{b}, *}$, PATRICK CAÑADAS ${ }^{\mathrm{b}, \mathrm{c}}$ and PATRICK CHABRAND ${ }^{\mathrm{a}}$
}

\begin{abstract}
${ }^{\mathrm{a}}$ CNRS UPR 7051, Laboratoire de Mécanique et d'Acoustique, Marseille, France; ${ }^{\mathrm{b}}$ CNRS UMR 7052, Laboratoire de Biomécanique et Biomatériaux Ostéo-Articulaires, Créteil, France; ${ }^{\mathrm{C}}$ INSERM UMR 492 Fonctions Cellulaire et Moléculaire de l'Appareil Respiratoire et des Vaisseaux, Créteil, France

The control of many cell functions including growth, migration and mechanotransduction, depends crucially on stress-induced mechanical changes in cell shape and cytoskeleton (CSK) structure. Quantitative studies have been carried out on 6-bar tensegrity models to analyse several mechanical parameters involved in the mechanical responses of adherent cells (i.e. strain hardening, internal stress and scale effects). In the present study, we attempt to generalize some characteristic mechanical laws governing spherical tensegrity structures, with a view of evaluating the mechanical behaviour of the hierarchical multi-modular CSK-structure. The numerical results obtained by studying four different tensegrity models are presented in terms of power laws and point to the existence of unique and constant relationships between the overall structural stiffness and the local properties (length, number and internal stress) of the constitutive components.
\end{abstract}

Keywords: Multi-modular cytoskeleton; Elasticity modulus; Pre-stress; DCSA-filaments

\section{INTRODUCTION}

Mechanical cellular responses play a fundamental role in tissue development and adaptation: for example, bone modelling and remodelling involve a multi-step process of cellular mechanotransduction. During these processes, bone cells detect mechanical signals which are integrated and result in the occurrence of appropriate changes in the bone architecture [19]. At the cellular scale, the mechanical forces exerted through the extracellular matrix are balanced by internal forces exerted by the cytoskeleton (CSK) [6]. This force balance appears to be crucial to many cell functions such as the control of cell shape, growth and differentiation [7,8,21]. Basically, cell deformability and mechanical responses depend on several interactive processes between factors such as the structural CSK-architecture and cell/cell and cell/extracellular matrix attachment. Moreover, there exists a growing amount of experimental evidence that the main cellular functions are controlled via multiple transmission pathways involving a hierarchical organisation of multimodular CSK substructures from the molecular to the overall cellular scale. Each of these substructures is regulated by specific mechanical responses associated to specific biological functions [5,7,9,13,22,23].

Various models for the CSK based on structural mechanics have been proposed to describe the relationships between external and internal forces as well as between cell deformation and stiffness. Satcher and Dewey [16] have estimated the mechanical response of the endothelial cell CSK, theoretically, based on the distributed cytoplasmic structural actin (DCSA) alone, without taking the other CSK filamentous networks (e.g. stress fibres, microtubules and intermediate filaments) into account. In this study using the classical foam theory, the authors suggested that the overall cell deformation might result from the bending of individual DCSA-filaments in response to the mechanical stresses exerted on the cell. However, this foam model takes into account neither the cellular prestress nor the specific mechanical and geometrical properties of the various types of CSKfilaments. Some recently developed structural models based on the tensegrity concept have several features which are consistent with the mechanical behaviour observed in adherent cells, e.g. the strain-hardening and the prestress dependency of the cells' mechanical responses. These complex mechanical responses have been closely correlated with the mechanical properties of the spatial CSK-filamentous network and with its structural reorganization $[2,4,17,28,30]$.

Tensegrity structures develop an intrinsic stabilising tension balanced by compression, which is called the prestress. When subjected to a local mechanical stress, the constitutive components of the tensegrity structures undergo a complete spatial reorganization and thus maintain a balance between the internal and external

\footnotetext{
*Corresponding author.
} 
forces. A tensegrity model has been proposed by Ingber [7] to account for the deformability of adherent cells, taking the CSK to be a network of interconnected stressbearing components (e.g. actin filaments, stress fibres) and isolated compression-bearing components (e.g. microtubules associated with intermediate filaments). Quantitative analytical and numerical studies based on this hypothesis were carried out on a 6-bar tensegrity structure with a view of describing the non linear stress-strain relationships and the scale effects as well as the internal stress dependence of the overall stiffness of in vitro endothelial cell cultures $[2,18,20,30]$. However, it has not been established so far (i) whether this 6-bar tensegrity model is representative of tensegrity structures in general or (ii) how more complex tensegrity structures could be used to predict the mechanical behaviour of the cells, taking into account the hierarchical organisation of the multi-modular assemblies which constitute the CSKstructure.

The aim of the present study is precisely to define general mechanical laws which are applicable to several spherical tensegrity structures and could be used to determine the mechanical behaviour of various still unknown CSK patterns. For this purpose, we analysed the influence of some local characteristic parameters such as the internal tension, the scale and the number of constitutive components on the overall elasticity modulus of various spherical tensegrity structures, using two models that differ from both the numerical and mechanical points of view. The differences observed are analysed and the biological relevance of the respective models is discussed.

\section{METHOD}

\section{Theoretical Tensegrity Model}

The four elementary tensegrity structures numerically tested are spatial systems which are arranged in apparently spherical patterns and are composed of a discontinuous network of quasi-rigid bars compressed by a continuous network of pre-stretched elastic cables. The so-called simplex, quadruplex, octahedron and cuboctahedron are spherical tensegrity structures composed of 3 bars and 9 cables, 4 bars and 12 cables, 6 bars and 24 cables and 12 bars and 48 cables, respectively (see Fig. 1). The constitutive cables and bars are defined in terms of their geometrical characteristics (length $L_{\mathrm{c}}, L_{\mathrm{b}}$; cross-sectional area $S_{\mathrm{c}}, S_{\mathrm{b}}$ ) and their mechanical properties (Young's modulus $\left.E_{\mathrm{c}}, E_{\mathrm{b}}\right)$. Each elastic cable behaves like a linear extension spring which is initially stretched in the reference state of the structure (i.e. when no external forces are applied to the overall structure). The particularity of the elastic cable is its unilateral mechanical behaviour, i.e. the cable loses its rigidity whenever its actual length $L_{\mathrm{c}}$ becomes smaller than the resting length $L_{\mathrm{oc}}$. The resulting stretching force $T_{\mathrm{c}}$ in the cable is defined as follows:

$$
\left\{\begin{array}{l}
T_{\mathrm{c}}=\frac{(\mathrm{ES})_{\mathrm{c}}}{L_{\mathrm{oc}}}\left(L_{\mathrm{c}}-L_{\mathrm{c}}^{(\mathrm{r})}\right)+T_{\mathrm{c}}^{(\mathrm{r})} ; \quad T_{\mathrm{c}}>0 \\
T_{\mathrm{c}}=0 \text { then } L_{\mathrm{c}}<L_{\mathrm{oc}}
\end{array}\right.
$$

Let $T_{\mathrm{c}}^{(\mathrm{r})}$ be the positive stretching force in the reference state defined by $T_{\mathrm{c}}^{(\mathrm{r})}=(E S)_{\mathrm{c}}\left(L_{\mathrm{c}}^{(\mathrm{r})}-L_{\mathrm{oc}}\right) /\left(L_{\mathrm{oc}}\right)$ and $L_{\mathrm{oc}}$ the length of the unloaded cable (see Fig. 2A).

The mechanical behaviour of the bars is similar to that of a classical spring and the compression force $T_{\mathrm{b}}$ in the bar is defined as follows:

$$
T_{\mathrm{b}}=\frac{(\mathrm{ES})_{\mathrm{b}}}{L_{\mathrm{ob}}}\left(L_{\mathrm{b}}-L_{\mathrm{b}}^{(\mathrm{r})}\right)+T_{\mathrm{b}}^{(\mathrm{r})}
$$

Let $T_{\mathrm{b}}^{(\mathrm{r})}$ be the compression force in the reference state $T_{\mathrm{b}}^{(\mathrm{r})}=(\mathrm{ES})_{\mathrm{b}}\left(\left(L_{\mathrm{b}}^{(\mathrm{r})}-L_{\mathrm{ob}}\right) / L_{\mathrm{ob}}\right)$ and $L_{\mathrm{ob}}$ be the length of the unloaded bar (see Fig. 2B). The cross-sectional area and the Young's modulus of both components are assumed to be constant. In the reference state, the stable shape of the tensegrity structure studied depends on the equilibrium between the tension in the cables $T_{\mathrm{c}}^{(\mathrm{r})}$ and $T_{\mathrm{b}}^{(\mathrm{r})}$ the compression in the bars.

\section{Study Conditions and Constitutive Equations}

We analysed the overall deformation of the four spherical tensegrity structures (simplex, quadruplex, octahedron and cuboctahedron ) subjected to extension-compression as the result of the forces applied at the three (four in the case of the quadruplex) nodes (extremities of the bars) lying in the upper plane. The structures were all assumed to be fixed to a rigid support by spherical joints at the three

A)

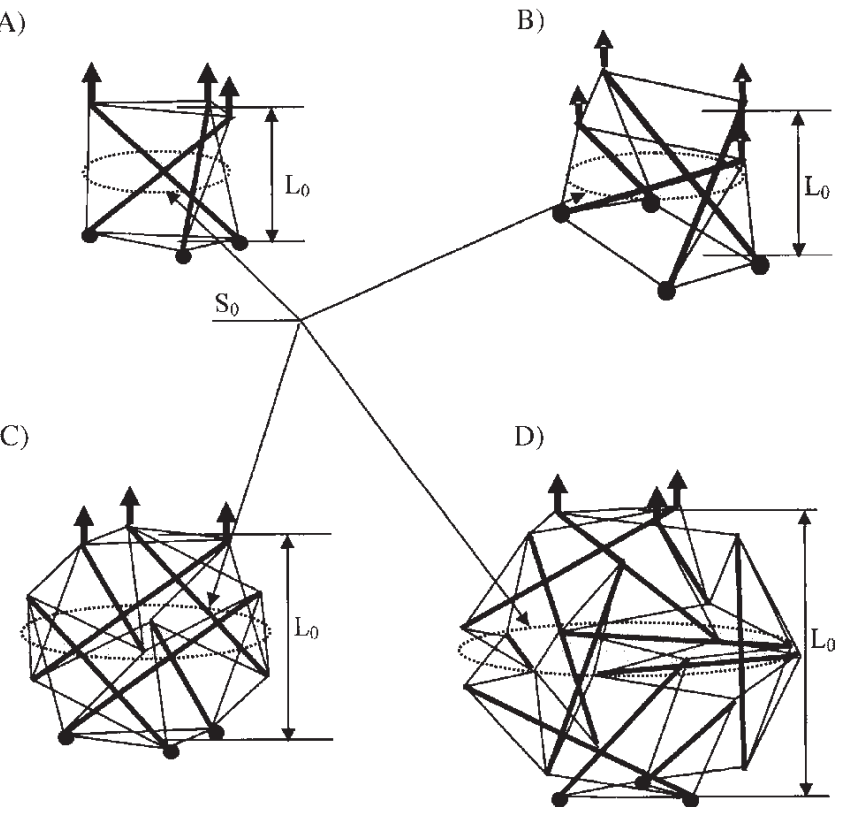

FIGURE 1 Four types of spherical tensegrity structures subjected to extension forces: (A) Simplex: 3 bars and 9 cables; (B) Quadruplex: 4 bars and 12 cables; (C) Octahedron: 6 bars and 24 cables and (D) Cuboctahedron: 12 bars and 48 cables. 
A)

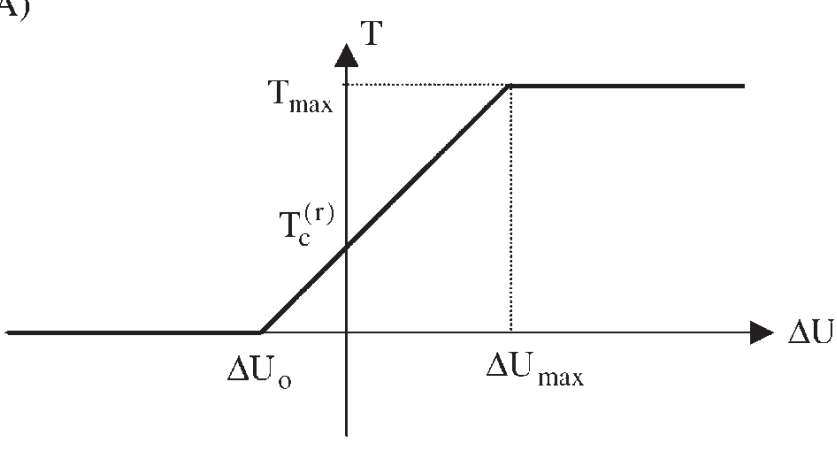

B)

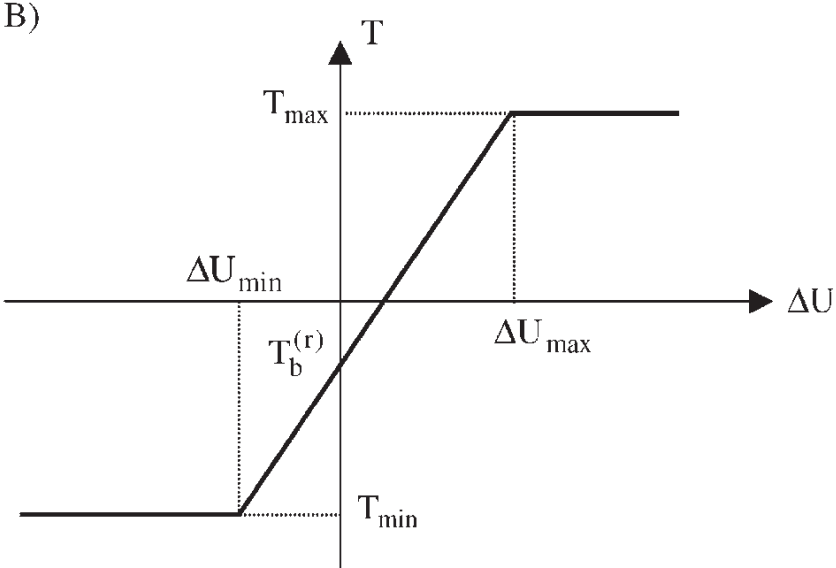

FIGURE 2 Definition of the constitutive equations of (A) the cable under unilateral conditions $\left(T_{c}=0\right.$ then $\left.\Delta U \leq \Delta U_{\mathrm{o}}\right)$ and (B) the bar. $\Delta U$ gives the relative elongation $\left(L_{\mathrm{p}}-L_{\mathrm{op}}\right)$ of the components $\mathrm{p}$ (bar or cable). By convention, $T_{\mathrm{b}}$ is taken to be negative and $T_{\mathrm{c}}$ is taken to be positive.

lower nodes (four nodes in the case of the quadruplex) (see Fig. 1). In both models herein developed, an incremental method was used to calculate the nodal displacements of the tensegrity model subjected to external forces. This method consists of applying an incremental load and calculating a new equilibrium configuration of the tensegrity structure at each loading step. It was assumed that the successive states of deformation were different from the reference state (that which existed prior to the loading). The problem to be solved is non linear because of the finite deformation and the non linear "displacement-strain" relationship between the constitutive components.

The first model, which is based on a Finite Element Method (FEM), consists to solve the following non linear system of equations under quasi static conditions:

$$
\{\mathbf{F}\}=[K(\mathbf{u})]\{\mathbf{u}\}
$$

Equation (3) describes the equilibrium between internal and external forces applied to the nodes, using the specific behavioural laws [see Eqs. (1) and (2)] governing each constitutive element (bars and cables). The components of the column vector $\{\mathbf{F}\}$ are the external forces applied to the nodes of the structure in the three spatial directions.
The components of the unknown column vector $\{\mathbf{u}\}$ are the resulting nodal displacements in the three directions. The matrix $[K(\mathbf{u})]$, which is the so-called rigidity matrix of the overall tensegrity structure, is updated at each loading step. Due to the FEM used, the computation failed as soon as the tension in one cable dropped to zero [see Eq. (1)].

To avoid this problem, we then used the second model, based on the Non Smooth Contact Dynamics (NSCD) Method which was developed to study short contact dynamic problems [10]. In this model, the tensegrity structures are taken to be a set of isolated material points that are connected to each other by so-called interaction laws. These material points are the nodes of the structure which are assumed to have a non zero mass. The interaction laws between the material nodes describe the geometrical and mechanical properties of the components (bars and cables) as well as the unilateral mechanical behaviour of the elastic cables (see Fig. 2). The overall deformation of the tensegrity structure is deduced by solving the following system of equations under dynamic conditions:

$$
[M(\mathbf{u})]\{\ddot{\boldsymbol{u}}\}=\{\mathbf{F}\}+\{\mathbf{r}(\mathbf{u})\}
$$

where $[M(\mathbf{u})]$ is the mass matrix of the overall structure (material points of the structure) and $\{\mathbf{r}(\mathbf{u})\}$ the column vector of the interaction forces between the material nodes satisfying unilateral conditions as far as the cables are concerned.

\section{Definition of the Tensegrity Model Stiffness}

The tensegrity model response was analysed in terms of the apparent elasticity modulus deduced from the overall stress-strain relationship. The equivalent stress $\sigma$ was defined by the ratio between the norm of the external force vector and the mean cross-sectional area $S_{0}$ of the overall structure:

$$
\sigma=\frac{\|\{\mathbf{F}\}\|}{S_{0}}
$$

$S_{0}$ corresponds to the area of a circumscribed circle which is normal to the direction of the external forces applied and located in the intermediate plane of the structure in the reference state (Fig. 1).

The apparent strain $\varepsilon$ of the overall structure was defined along the loading axis as follows:

$$
\varepsilon=\frac{\Delta L}{L_{0}}
$$

where $L_{0}$ is the distance between the lower and upper planes in the reference state (Fig. 1). The apparent elasticity modulus $E$ of the tensegrity model was defined by the stress/strain ratio at $10 \%$ deformation of the whole structure. 


\section{Non Dimensional Parameters of the Tensegrity Model}

The tensegrity model is characterised by (i) the length of the constitutive bars $\left(L_{\mathrm{b}}^{(\mathrm{r})}\right)$ which defines the overall structural scale, (ii) the internal tension defined by the initial cable tension $T_{\mathrm{c}}^{(\mathrm{r})}$ as well as by (iii) the number of bars $(N)$. In order to analyse the overall elasticity modulus given by the tensegrity model at the cellular level, the bar length $L_{\mathrm{b}}^{(\mathrm{r})}$, the pre-stretching force $T_{\mathrm{c}}^{(\mathrm{r})}$ of the tensegrity structures in the reference state and the apparent elasticity modulus $E$ were normalised using two constant material characteristics, namely the Young's modulus $E_{\mathrm{c}}$ and the radius $r_{\mathrm{c}}$ of the cable.

$$
\begin{gathered}
T^{*}=\frac{T_{\mathrm{c}}^{(\mathrm{r})}}{E_{\mathrm{c}} S_{\mathrm{c}}} \\
L^{*}=\frac{L_{\mathrm{b}}^{(\mathrm{r})}}{r_{\mathrm{c}}} \\
E^{*}=\frac{E}{E_{\mathrm{c}}} .
\end{gathered}
$$

The normalised overall elasticity modulus $E^{*}$ was expressed as a function of the normalised elastic tension $T^{*}$, the normalised length $L^{*}$ [Eqs. (7) and (8)] and the number of constitutive components (bars) $N$, thus yielding an expression for the proportional overall structural stiffness in relation to the properties of the individual components.

\section{RESULTS}

The normalised elasticity modulus given by the numerical solution of the constitutive equations obtained in the framework of both the non smooth contact dynamic model and the finite element model are presented in Figs. 3-5 as a function of three main parameters of the tensegrity structures, i.e. the normalised length of the constitutive bars $L^{*}$, which is characteristic of the size of the structure, the normalised internal tension $T^{*}$, which corresponds to the initial extension of the cables in the reference state and the number of bars $N$, which differs between the four tensegrity structures (simplex, octahedron, quadruplex and cuboctahedron) tested.

Figure 3 gives the $\left(E^{*}-T^{*}\right)$ relationships curve-fitted by a power-law equation on the logarithmic scale. The physical properties obtained (length, radius and Young's modulus of the components) are similar with all four tensegrity structures tested. The structural stiffness increases with the internal stress; i.e. the slope of the curve was +0.4 with the octahedron (Fig. 3C), +0.54 with the simplex (Fig. 3A), +0.67 with the cuboctahedron (Fig. 3D) and +0.79 with the quadruplex (Fig. 3B). However, the slopes of the logarithmic $\left(E^{*}-T^{*}\right)$ curves vary non linearly with the tensegrity structure (i.e. with the number of bars, $N$ ). To explain these discrepancies, we analysed the influence of the number of constitutive bars on the overall structural stiffness. It can be seen from Fig. 4 that a decrease in the overall normalised elasticity modulus occurred when the number of bars increased. It is worth noting that regardless of $L^{*}, E^{*}$ was found to exhibit a negative dependence on $N$ (i.e. the slope $\approx-2.7$ on the $\log E^{*}-\log N$ curve, see Fig. 4). This result means that increasing the number of interconnected components in a spherical tensegrity structure tends to soften the structure. In addition, we noted that the normalised apparent elasticity modulus $E^{*}$ decreases proportionally to the inverse of the square of $L^{*}$ (slope -2 of the curve) in all the tensegrity structures studied, i.e. whatever the number of constitutive components. In other words, the structural stiffness decreases as the size of the structures tested increases. A similar $L^{*}{ }^{-2}$-dependency was previously observed with a 16-component tensegrity model [29] and when the octahedron was subjected to other conditions of attachment to a rigid base and external forces of other kinds were applied [2,28].

Based on all these dependencies on the overall elasticity modulus of the tensegrity structures tested, we deduced the following unique non dimensional parameter:

$$
R=\frac{\left(T^{*}\right)^{0,5}}{N^{2,7} \cdot\left(L^{*}\right)^{2}}
$$

The parameter $R$ was determined and used to estimate the normalised elasticity modulus $E^{*}$ of a large variety of tensegrity structures defined in terms of the triplex $\left(L^{*}, T^{*}\right.$, $N$ ). It is worth mentioning that the stiffness of the tensegrity structure could be balanced by the number of constitutive components $(N)$ and the size of the structure (component length $L^{*}$ ). A large number of short components yields approximately the same structural apparent elasticity modulus. In addition, we have observed for the four types of spherical tensegrity structures tested that the elasticity modulus in the perpendicular direction (i.e. apparent shear modulus) is not equivalent to $1 / 3$ of the apparent elasticity modulus, ratio which is characteristic of an equivalent isotropic elastic medium (results not shown). A more detailed study has already been performed on the octahedron model (see Ref. [28]). Moreover, in a recent study, we have shown that the Poisson's ratio of a highly deformed 30-element tensegrity model is not constant and always greater than the standard Poisson's ratio $\nu$ of a continuous medium, i.e. $\nu>0.5$ (see Ref. [2]).

\section{DISCUSSION}

\section{Multi Factorial Mechanical Behaviour of Tensegrity Structures}

The present study was the first attempt made so far to generalise the laws governing the relationships between the elasticity modulus $\left(E^{*}\right)$ with which the tensegrity cell 
A) SIMPLEX

4
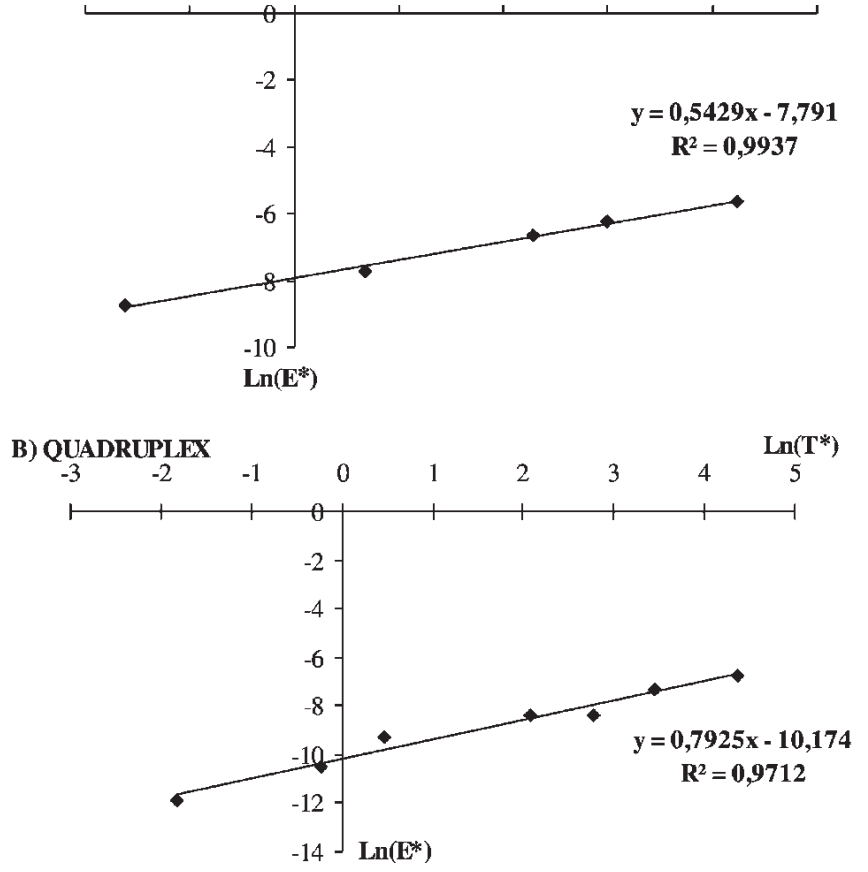

C) OCTAHFDRON

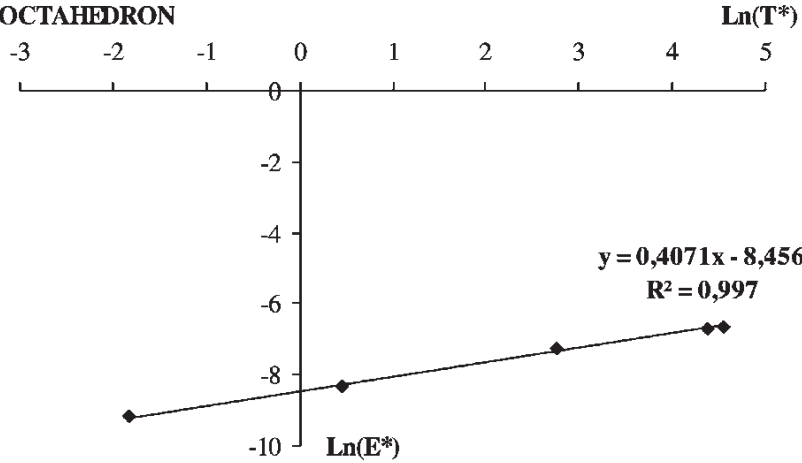

D) CUBOCTAHEDRON

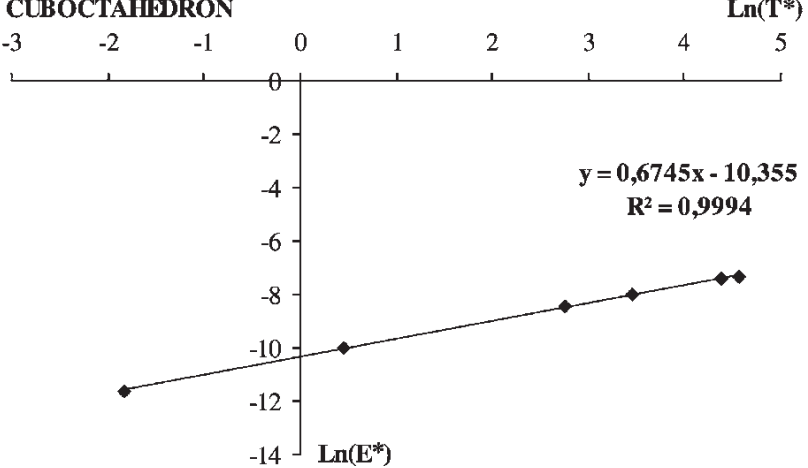

FIGURE 3 Normalised elasticity modulus $E^{*}$ of (A) the simplex ( 3 bars), (B) the quadruplex (4 bars), (C) the octahedron (6 bars) and (D) the cuboctahedron (12 bars) subjected to extension, plotted versus the normalised internal tension $T *$. The linear equation was obtained from the curve-fitting procedure performed with the numerical results obtained with each type of tensegrity structure.

model deals and local properties such as (i) the internal tension $T^{*}$, which corresponds to the cable strain in the reference state (before loading), (ii) the number of constitutive components $N$ and (iii) the normalised component length $L^{*}$, which characterises the size of the overall structure. The use of non dimensional expressions

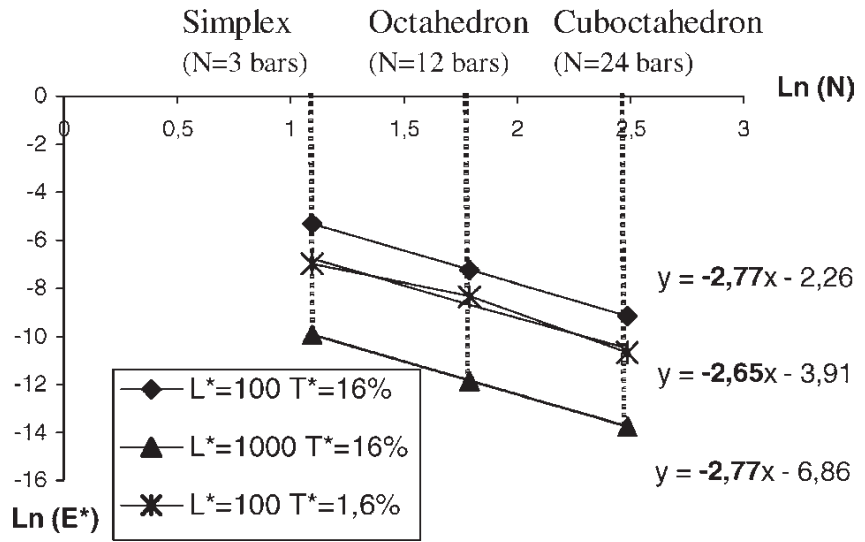

FIGURE 4 Numerical relationships between the normalised elasticity modulus and the number of elements on the logarithmic scale, based on three different pairs of values (the normalised length $L *$ and the normalised internal tension $T *$ ).

to describe the mechanical response of the tensegrity model makes it possible to show the existence of a constant proportionality between the components and the overall mechanical properties. In fact, the mechanical behaviour of tensegrity structures is multi factorial and depends strongly on the local physical properties (internal tension, size, number of components, etc.) as well as on the type of nodal attachment to the rigid support and the external loading conditions. It was observed that increasing the number and increasing the length of the constitutive components both soften the spherical tensegrity model to a similar extent (slope -2 of the $\left(\log E^{*}-\log L^{*}\right)$ curve versus slope -2.7 of the $\left(\log E^{*}-\log N\right)$ curve); whereas the four tensegrity models tested become stiffer when the internal tension is increased. These dependencies could be expressed in a single constant number $R$ [see Eq. (10)], which may be representative of a large variety of spherical tensegrity structures. The mechanical behaviour of more complex structures (e.g. those having a larger number of components, greater internal tension, etc.) is therefore comparable to that of an elementary simplified tensegrity structure with a similar number $R$. This number could be defined by several triplexes of the three main parameters

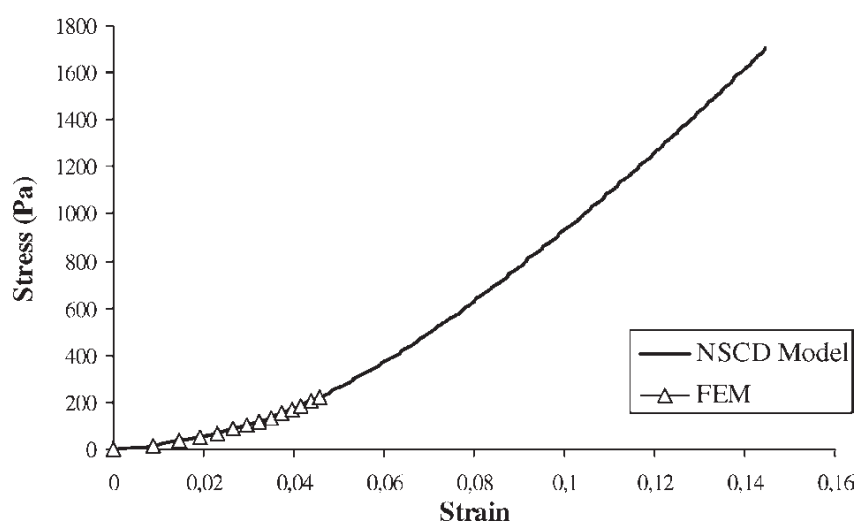

FIGURE 5 Numerical "stress-strain" relationships obtained on a 30 -element tensegrity structure $\left(L^{*}=100, T^{*}=0.01 \%\right)$ subjected to extension with the FEM and the NSCD Model. 
$\left(L^{*}, T^{*}, N\right)$. These results confirm the previously proposed hypothesis that simplified tensegrity structures may in fact be representative of more complex tensegrity structures [11]. These theoretical results also confirm the finding that the main features, such as the $L^{*}$ and $T *$ effects of the various tensegrity models studied under several attachment and loading conditions do not differ fundamentally $[1,30]$.

\section{FEM versus NSCD Model}

The FEM based on a standard finite element analysis with bars and cables as the basic components, was used to study the mechanical behaviour of tensegrity structures. Although this model is easy to apply and involves fast computation rates, it can only be used for studying tensegrity structures under quasi static conditions with small deformations. Indeed, the FEM failed when one constitutive elastic cable was released during the overall deformation of the tensegrity structure tested. Moreover, in the case of tensegrity structures with a weak internal tension $T *$, the slackening of some constitutive elastic cables occurs when there is only a slight deformation of the overall tensegrity structure tested (see Fig. 5). To analyse tensegrity model deformations with a large range of $T^{*}(=[0.001-0.1])$, we therefore developed a numerical model in which the NSCD method was applied to the tensegrity concept, taking the unilateral mechanical behaviour of the elastic cable into account. The most original feature of this model is that it can be used in both static and dynamic studies on an infinite range of tensegrity structures involving various spatial distributions of the constitutive components. However, since the number and the spatial organization of the constitutive components are unlimited, there are still many difficulties to be overcome before we can define and develop a more complex structure in the framework of the tensegrity structure concept.

\section{Biological Interpretation}

The cellular overall deformation is closely related to the microenvironmental mechanical conditions (e.g. external applied stress, cell/cell or cell/extracellular matrix attachment conditions) and results from a spatial rearrangement of the CSK filamentous network [21,24]. Moreover, many authors have explained in terms of their common tensegrity nature, the prestress and the non linear responses (stress-hardening) observed in the CSK of living adherent cells $[12,24,25]$. Authors of previous studies on tensegrity structures have established that living cells and tensegrity models interestingly share a great number of common structural and mechanical features $[4,18,26,27]$.

More recently, authors have shown the heterogeneity of the mechanical properties at work within the cytoplasm of living adherent cells, stressing the need to perform multimodular analyses on the mechanical cell responses
[13,15,31]. In particular, Laurent et al. have performed 3D-reconstructions of low and high density actin filament networks which revealed two compartments; (i) the cortical actin network constitutes a thin mantle everywhere in the cell and may contain a large number of short, low-density actin filaments, (ii) the cytosolic actin network is organised in large stress fibres through the whole cell coupled to other highly polymerised CSKfilaments (i.e. the microtubules associated to the intermediate filaments) and may contain a smaller number of high-density, thick, long F-actin stress fibers [13]. The cortical actin network is the faster, softer, more fluidified compartment and the cytosolic actin network which is responsible for the stability of the cell and its anchorage to the substrate via a process of focal adhesion, is a slower, stiffer, more solidified compartment [3,13]. Moreover, bead twisting measurements on living adherent cells showed that the CSK response may be advantageously partitioned into a slightly tensed, easily deformable cortical CSK compartment and a highly tensed, largely rigid 3D-internal cytosolic CSK network [14]. In addition, these two compartments, especially the cytosolic one, exhibit a stress-hardening response. The cellular $L^{*}$ and $N$ values obtained in our theoretical studies on tensegrity structures confirm the hypothesis put forward by Laurent et al. [13] that the normalised constitutive length $L *$ is 1 order of magnitude smaller and the number of components $N$ about 2 orders of magnitude greater in cortical than in cytosolic CSK microstructures. The structural stiffness of cortical CSK is probably lower than that of the cytosolic CSK, as observed experimentally. Moreover, the tensegrity stiffness $\left(E^{*}\right)$ was found to increase slightly with the internal tension $(T *)$ in all four models. A rough estimate of the prestress values in elastic fibres and filaments in each of the two CSK compartment suggests that the $T *$ values may be about 2 orders of magnitude smaller in the cortical than the cytosolic CSK, which would also contribute to decreasing the structural cortical stiffness.

Although tensegrity models are still greatly oversimplified in comparison with biological reality, the tensegrity concept, nevertheless, provides a useful approach for investigating how each of the CSK substructures located at different points in the cytoplasm and/or at various scales may be involved in the mechanical responses of living cells during biological processes.

\section{Acknowledgements}

We are indebted to Professor D. Isabey and Dr V.M. Laurent (INSERM UMR 492 Fonctions Cellulaire et Moléculaire de l'Appareil Respiratoire et des Vaisseaux, Créteil, France, Cellular Biophysics and Biomechanics Laboratory, Swiss Federal Institute of Technology.) Professor M. Jean and M. Villautreix for their contributions. This study was supported by Centre National pour la Recherche Scientifique (Programme Matériaux, 2001). 


\section{References}

[1] Cañadas, P., et al. (2000) "Time response of a viscoelastic tensegrity model to approach cell dynamical response", Archives of Physiology and Biochemistry 108(1/2), 170.

[2] Cañadas, P., et al. (2002) "A viscoelastic cellular tensegrity model to analyze the cytoskeleton mechanical response", Journal of Theoretical Biology 218, 155-173.

[3] Choquet, D., Felsenfeld, D.P. and Sheetz, M.P. (1997) "Extracellular matrix rigidity causes strengthening of integrin-cytoskeleton linkages", Cell 88, 39-48.

[4] Coughlin, M.F. and Stamenovic, D. (1998) "A tensegrity model of the cytoskeleton in spread and round cells", Journal of Biomechanical Engineering 120, 770-777.

[5] Forgacs, G. (1995) "On the possible role of cytoskeletal filamentous networks in intracellular signaling: an approach based on percolation", Journal of Cell Science 108, 2131-2143.

[6] Harris, A.K., Wild, P. and Stopak, D. (1980) "Silicone rubber substrata: a new wrinkle in the study of cell locomotion", Science 208(11), 177-179.

[7] Ingber, D.E. (1997) "Tensegrity: the architectural basis of cellular mechanotransduction", Annual Review of Pysiology 59, 575-599.

[8] Ingber, D.E. (1998) "Tensegrity and the emergence of a cellular biophysics", In: Beysens, D. and Forgacs, G., eds, Dynamical Networks in Physics and Biology (EDP Sciences/Springer-Verlag, France/Berlin).

[9] Ingber, D.E., et al. (2000) "Opposing views on tensegrity as a structural framework for understanding cell mechanics", Journal of Applied Physiology 89, 1663-1678.

[10] Jean, M. (1999) "The non-smooth contact dynamics method", Computer Methods in Applied Mechanics and Engineering 177, $235-257$.

[11] Kebiche, K., Kazi-AAoual, M.N. and Motro, R. (1999) "Geometrical non linear analysis of tensegrity systems", Engineering Structures 21, 864-876.

[12] Laurent, V., et al. (1999) "Role of prestress on cytoskeleton stiffness analyzed by tensegrity model", Archives of Physiology and Biochemistry 107, 54.

[13] Laurent, V.M., et al. (2002) "Tensegrity behavior of cortical and cytosolic cytoskeletal components in twisted living adherent cells", Acta Biotheoretica, 50(4), 331-356.

[14] Laurent, V.M., et al. (2002) "Assessment of mechanical properties of adherent living cells by bead micromanipulation: comparison of magnetic twisting cytometry vs optical tweezers", Journal of Biomechanical Engineering, 124, 908-921.

[15] Mathur, A.B., Truskey, G.A. and Reichert, W.M. (2000) "Atomic force and total reflection fluorescence microscopy for the study of force transmission in endothelial cells", Biophysical Journal 78, $1725-1735$.

[16] Satcher, R.L. and Dewey, C.F. (1996) “Theoretical Estimates of Mechanical Properties of the Endothelial Cell Cytoskeleton", Biophysical Journal 71, 109-118.

[17] Stamenovic, D. and Coughlin, M.F. (1999) "The role of prestress and architecture of the cytoskeleton and deformability of cytoskeletal filaments in mechanics of adherent cells: a quantitative analysis", Journal of Theoretical Biology 201, 63-74.

[18] Stamenovic, D. and Coughlin, M.F. (2000) "A quantitative model of cellular elasticity based on tensegrity", Journal of Biomechanical Engineering 122, 39-43.

[19] Turner, C.H. and Pavalko, F.M. (1998) "Mechanotransduction and functional response of the skeleton to physical stress: the mechanisms and mechanics of bone adaptation", Journal of Orthopaedic Science 3, 346-355.

[20] Volokh, K.Y., Vilnay, O. and Belsky, M. (2000) "Tensegrity architecture explains linear stiffening and predicts softening of living cells", Journal of Biomechanics 33, 1543-1549.

[21] Wang, H., et al. (1995) "Cell orientation response to cyclically deformed substrates: experimental validation of a cell model", Journal of Biomechanics 28(12), 1543-1552.

[22] Wang, N. (1998) "Mechanical interactions among cytoskeletal filaments", Hypertension 32, 162-165.

[23] Wang, N., Butler, J. and Ingber, D. (1993) "Mechanotransduction across the cell surface and through the cytoskeleton", Science $\mathbf{2 6 0}$, $1124-1127$

[24] Wang, N. and Ingber, D.E. (1994) "Control of cytoskeletal mechanics by extracellular matrix. Cell shape and mechanical tension”, Biophysical Journal 66, 1-9.
[25] Wang, N., et al. (2001) "Mechanical behavior in living cells consistent with the tensegrity model", Biophysics 98(14), 7765-7770.

[26] Wendling, S., et al. (2000) "Tensegrity model to describe the coupling between elastic energy and deformation of cells twisted by magnetic probe", Archives of Physiology and Biochemistry 108(1/2), 9 .

[27] Wendling, S., et al. (2002) "Interrelations between elastic energy and strain in a tensegrity model; contribution to the analysis on the mechanical response in living cells", Computer Methods in Biomechanics and Biomedical Engineering, 5(1), 1-6.

[28] Wendling, S., Oddou, C. and Isabey, D. (1999) "Stiffening response of a cellular tensegrity model", Journal of Theoretical Biology 196(3), 309-325.

[29] Wendling, S., Oddou, C. and Isabey, D. (2000) "Approche structurale de la mécanique du cytosquelette: solide alvéolaire vs modèle de tenségrité", C.R. Acad. Sci. Série. IIb(328), 97-104.

[30] Wendling, S., et al. (2000) "Role of cellular tone and microenvironmental conditions on cytoskeleton stiffness assessed by tensegrity model", Eur. Phys. J. AP 9, 51-62.

[31] Yamada, S., Wirtz, D. and Kuo, S.C. (2000) "Mechanics of living cells measured by laser tracking microrheology", Biophysical Journal 78, 1736-1747.

\section{APPENDIX}

The FEM consists to solve the non linear system of equations $(\{\mathbf{F}\}=[K(\mathbf{u})]\{\mathbf{u}\})$ under quasi static conditions using the quasi-static implicit Newton Raphson method of integration (Standard quasi-static implicit version of ABAQUS). The finite elements used to define the constitutive elements (bars and cables) of the tensegrity structure tested, are nonlinear axial springs. The constitutive equations of the elements (cables and bars) are done by the $\left(\Delta U-T_{\mathrm{p}}\right)$ relationships shown in Fig. 2 . $\Delta U$ gives the relative elongation $\left(L_{\mathrm{p}}-L_{\mathrm{op}}\right)$ of the components $\mathrm{p}$ (bar or cable) and $T$ the stretching or compression force as defined in Eqs. (1) and (2). By convention, $T_{\mathrm{b}}$ is taken to be negative and $T_{\mathrm{c}}$ is taken to be positive. It is to notice that the cables work only in extension and this unilateral condition is defined by $T_{\mathrm{c}}=0$ then $\Delta U \leq \Delta U_{\mathrm{o}}$ (with $\Delta U_{\mathrm{o}}$ the relative resting length of the cables before assembly in the structure). When the tensegrity structure is submitted to external applied stress, some cables become lack and the computation failed as soon as the tension in one cable dropped to zero due to the unilateral condition in the constitutive equation of the cables.

In the NSCD model, the constitutive elements of the tensegrity structure are considered as a set of material points which interact together. The interaction laws between the material nodes describe the geometrical and mechanical properties of the rigid bars as well as the geometrical and unilateral mechanical behaviour of the elastic cables. These interaction laws between specific material points are defined as follows:

$$
\left\{\begin{array}{c}
\bar{u} \geq 0 \\
R \geq 0 \\
\bar{u} \cdot R=0
\end{array}\right.
$$


with

$$
\left\{\begin{array}{l}
u=l-l_{0} \\
\bar{u}=-u+\frac{1}{k} R
\end{array}\right.
$$

$l$ (respectively $l_{0}$ ) are the actual (respect. resting) length of the element; $k$, the rigidity of the element and $R$ the interaction force between neighboring points.
The unknowns of these conditions of complimentarily $(\bar{u} ; R)$ are taken into account in the problem [Eq. (4)] to resolve. This problem which is written at each increment of re-equilibrium in the form of a system of linear equations with constraints, could be directly resolved by algorithms used for unilateral contact problems. The details of the resolution methods of such problems are proposed in Ref. [10]. 